\title{
Foetal Alcohol Spectrum Disorder \\ in adopted children from East Europe countries in Spain
}

\author{
J. Clotet Caba ${ }^{1}$, A. Herranz Barbero ${ }^{1}$, C. Borras-Novell ${ }^{1}$, O. Garcia-Algar ${ }^{1}$, A. Bastons-Compta ${ }^{1}$, \\ M. Astals-Vizcaino ${ }^{1}$, V. Fumado², N. Gomez ${ }^{3}$ \\ ${ }^{1}$ GRIE, Neonatology Unit, Hospital Clinic-Maternitat, ICGON, IDIBAPS, BCNatal, Barcelona, Spain. ${ }^{2}$ Paediatrics Unit, \\ Hospital Sant Joan de Deu, Barcelona, Spain ${ }^{3}$ Psychiatry Unit, Hospital Vall Hebron, Barcelona, Spain
}

\section{INTRODUCTION}

Maternal alcohol consumption during pregnancy has long-lasting adverse effects, causing structural, behavioural, and cognitive damage despite a radically improved environment. Alcohol consumption during pregnancy has been unnoticed although the measurement through alternative biomarkers (neonatal meconium and maternal hair) rise to $65 \%$ of the target population ${ }^{1}$.

There are no official data about Foetal Alcohol Spectrum Disorder (FASD) in adopted children from these countries in Spain.

The aim was to investigate the frequency of FASD among children adopted from East Europe countries (Russia and Ukraine) in Barcelona (Spain).

${ }^{2}$ Total alcohol per capita consumption, 2016

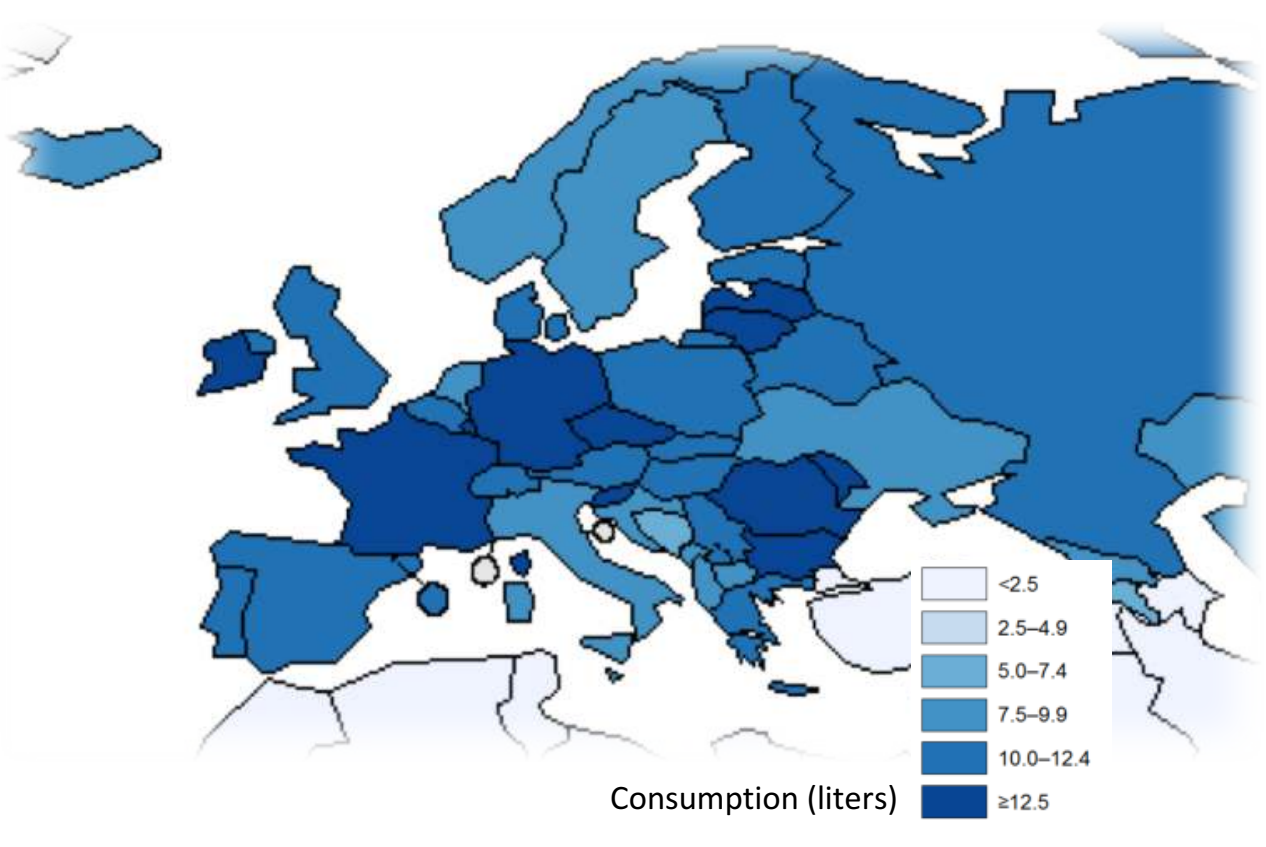

\section{MATERIAL/METHODS}

$>$ In a population-based, a prospective, observational, cross-sectional study was performed on 162 children between 8 and 24 years old and adopted from East Europe countries (Russia and Ukraine).

$>$ The children were examined by multidisciplinary team, at least 2 years after adoption, from paediatric, neuropsychological and somatometric perspectives, using 2016 Hoyme's criteria

International adoption (Catalonia, Spain), Countries of origin 2006-2014

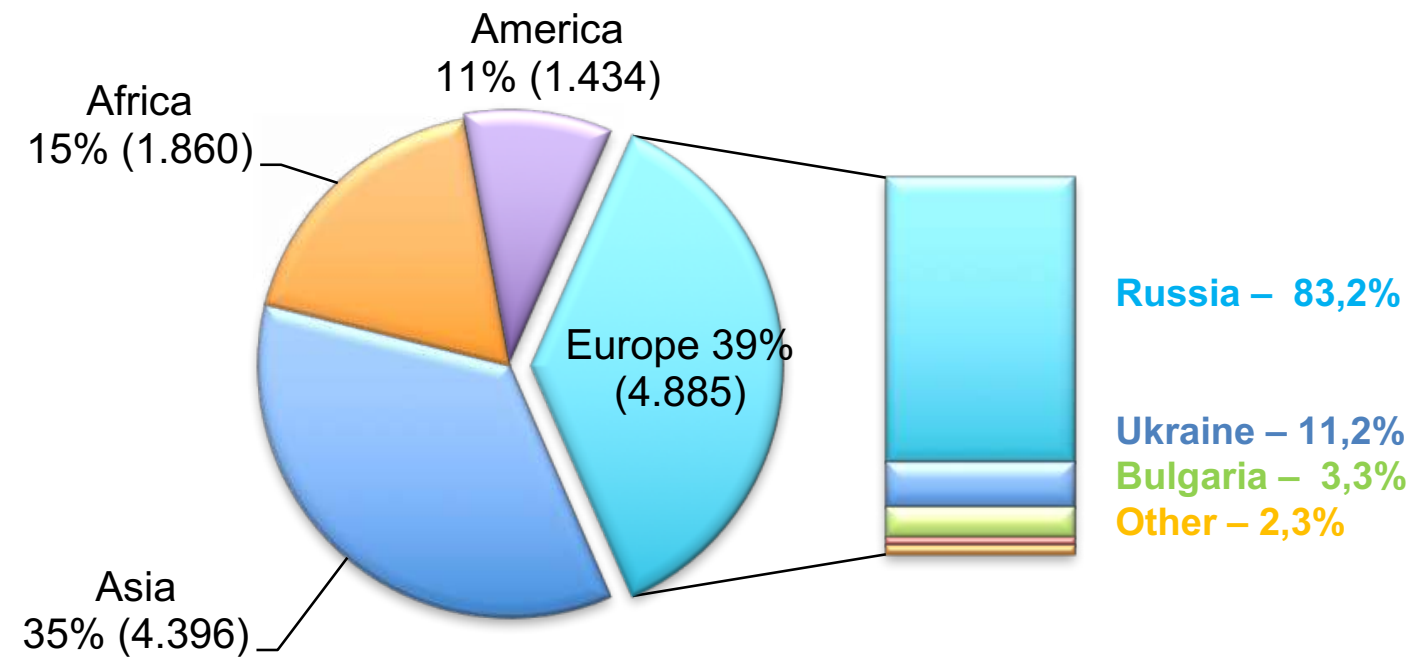

Diagnostic criteria (Hoyme, 2016)

- Confirmed consumption of alcohol during pregnancy

- Physical and somatometric assessment: weight, height, palpebral fissures, vermilion border of the upper lip, philtrum - Neuropsychological assessment: Cognitive impairment: global impairment, cognitive deficit Behavioural impairment

- Structural brain anomalies, recurrent nonfebrile seizures, specific major malformations

\section{RESULTS}

$>101$ boys $(62 \%)$ and 61 girls $(38 \%)$

$>$ Between 8 and 24 years of age (13,8 years)

$94 \%$ from Russia and $6 \%$ from Ukraine
Prevalence of FASD in adopted children

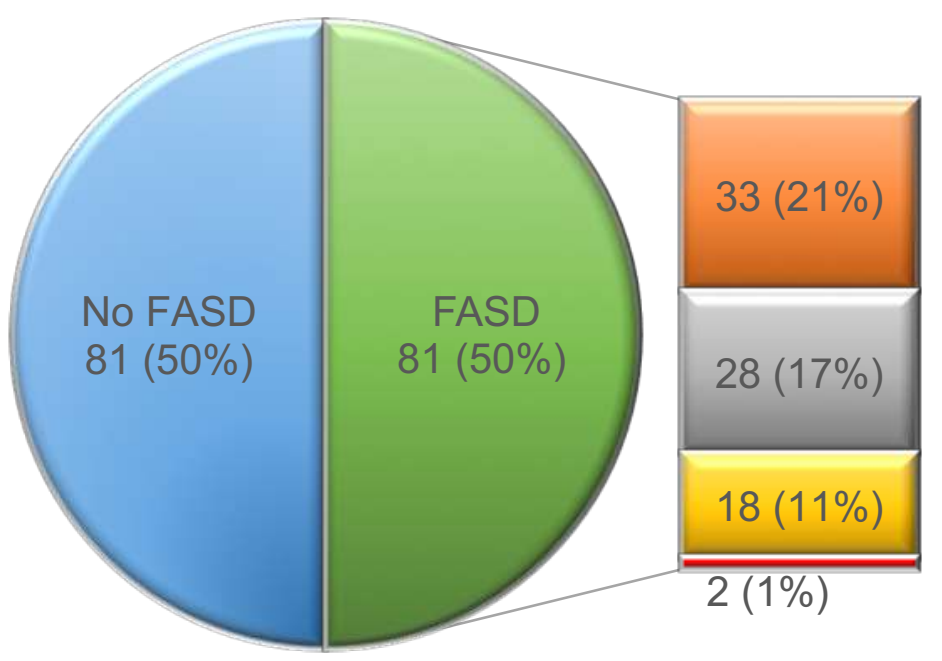

四No Foetal Alcochol Syndrom Disorder

回Foetal Alcochol Syndrom

四artial FAS

Alcohol-related neurodevelopmental disorders

IAlcohol-related birth defects

\section{CONCLUSIONS}

Foetal alcohol spectrum disorders were common in this long-term follow-up study of children adopted from orphanages in East Europe countries

$\checkmark$ An early diagnosis of FASD would benefit from interventions designed to meet the needs of FASD children

$\checkmark$ Adoptive parents and professionals need to be aware of the potential consequences of prenatal exposure to alcohol.

${ }^{1}$ Maternal hair testing to disclose self-misreporting in drinking and smoking behavior during pregnancy. Gomez-Roig MD. Alcohol, 2018

${ }^{2}$ Global status report on alcohol and health, WHO, 2018 